\title{
Perspective
}

Actualité en histoire de l'art

\section{L'histoire intérieure. Écrire une histoire du sida}

The Internal History. Writing the History of AIDS

Von Innen heraus - Vom Schreiben über AIDS

La storia interiore. Scrivere una storia dell'AIDS

La historia interior. Escribir una historia del sida

\section{Thibault Boulvain}

\section{(2) OpenEdition}

Journals

Édition électronique

URL : https://journals.openedition.org/perspective/7371

DOI : 10.4000/perspective.7371

ISSN : 2269-7721

Éditeur

Institut national d'histoire de l'art

Édition imprimée

Date de publication : 30 juin 2017

Pagination : 185-191

ISBN : 9782917902387

ISSN : $1777-7852$

Référence électronique

Thibault Boulvain, "L'histoire intérieure. Écrire une histoire du sida », Perspective [En ligne], 1 | 2017, mis en ligne le 31 décembre 2017, consulté le 16 janvier 2023. URL : http://journals.openedition.org/ perspective/7371; DOI : https://doi.org/10.4000/perspective.7371 


\section{L'histoire intérieure. Écrire une histoire du sida}

\author{
Thibault Boulvain
}

- Art AIDS America, 2015: Art AIDS America, Jonathan David Katz, Rock Hushka (dir.), cat. exp. (Tacoma, Tacoma Art Museum/Kennesaw, Zuckerman Museum of Art/New York, The Bronx Museum of the Arts/Chicago, Alphawood Gallery, 2015-2017), Tacoma/Washington/ Seattle, Tacoma Art Museum/University of Washington Press, 2015, 288 p., 200 ill. couleur.

- CRIMP, 1988 : Douglas Crimp (dir.), AIDS: Cultural Analysis/Cultural Activism, Cambridge, MIT Press, 1988, $272 \mathrm{p}$.

- Lebovici, 2017 : Élisabeth Lebovici, Ce que le sida m'a fait. Art et activisme à la fin $d u X X^{e}$ siècle, Zurich/Paris, JRPIRingier/La maison rouge - Fondation Antoine de Galbert (collection Lectures maison rouge), 2017, 320 p., 90 ill. nsb.

\section{Sismographie}

Des Cloches de la terre d'Alain Corbin, comme de ce type de livre qui a tout à voir avec l'histoire de son auteur, l'historien Michel Lagrée affirmait qu'il ne peut être écrit qu'à condition d'avoir, selon sa belle expression, des " archives intérieures ${ }^{1}$ ".

Celles-ci fournissent la matière archi-vivante du dernier ouvrage de la critique d'art et historienne de l'art Élisabeth Lebovici, Ce que le sida m'a fait. Art et activisme à la fin $d u X X^{e}$ siècle. "Un vaste corpus de matériaux, artefacts, affiches, flyers, cassettes audio et vidéo, archives personnelles ou officielles a nourri chaque chapitre. J'écris "nourri", je devrais peut-être dire "saturé". Car le problème aujourd'hui n'est pas de se confronter à la page blanche mais plutôt à sa propre tête, beaucoup trop remplie $^{2}$. " Comme ce "placard " d'où l'auteure sort, entre mille autres choses, du Fungizone, pour le traitement des candidoses : "Mes premiers rapports avec le sida " écrit-elle. "Cet épais liquide marron-orange que Patrick mettait dans sa bouche crevassée de blanc : les champignons. La langue de Francis, la langue de Philippe ${ }^{3}$." Patrick [Bracco], Francis, Philippe [Thomas] et les autres - beaucoup d'autres : tous habitent la vie de l'auteure, comme son ouvrage, qui, dans sa construction, en épouse le mouvement. Élisabeth Lebovici prend le lecteur à parti : " ce que je veux vous raconter, c'est comment j'ai été chamboulée par le Journal Annales de Lionel Soukaz ${ }^{4}$, écrit-elle au chapitre VIII qui lui est consacré. L'image vidéo, chez le cinéaste expérimental, "se fait le sismographe " d'un " chevauchement entre l'action politique et la vitalité des affects ${ }^{5}$ ". Auparavant, l'auteure s'est souvenue du "Time Square Show ", cette exposition mythique organisée à New York en juin 1980 par le Colab (Collaborative Projects, Inc.), un collectif d'artistes-et-activistes, dans un immeuble abandonné, au coin de la $41^{\mathrm{e}}$ Rue et de la $7^{\mathrm{e}}$ Avenue. Quelque chose s'est joué là aussi, pour elle : " Le Times Square Show a auguré de mon devenir-spectatrice, espérons-le, émancipée ${ }^{6}$. " Il l'a également poussée à changer de sujet de thèse, pour coller au pur présent : Thomas Blaikie, ce jardinier écossais de la fin du XVIII ${ }^{\mathrm{e}}$ siècle dont elle étudiait alors le journal, l'attend encore ; désormais, son doctorat portera plutôt sur " L'Argent dans le discours des artistes américains ${ }^{7}$. " Elle est arrivée à New York en 1979 pour suivre le cursus d'études curatoriales du Whitney Independent Study Program, et le prétexte est alors tout trouvé pour y rester trois ans. Le " Time Square Show " se voulait un " carrefour " de la société, comme la célèbre place à laquelle il empruntait son titre est réputée être " le carrefour du monde " : une ouverture du champ de l'art, une incitation à l'élargir : «l'exposition est aussi une place publique. Elle se veut réplique, au sens sismologique, d'un lieu parcouru par des énergies ${ }^{8}$ ".

Force est de le constater au fil des pages : Élisabeth Lebovici aime les tremblements, les vibrations, les secousses, ce(ux) qui les provoque(nt) comme ce(ux) qui les enregistre(nt), en art comme ailleurs dans la vie. Ainsi définit-elle dans son avant-propos l'activité de la critique d'art - qu'elle a commencé à pratiquer en pleine crise du sida comme une " sismographie ${ }^{9}$ ", cette attention constamment portée au soubresaut, au vacillement. On se souvient qu'en mai 2011, au Centre Pompidou, elle nous incitait d'ailleurs à prêter attention, en histoire de l'art, au "phénomène de contretemps : pas une réaction, ou pas seulement une réaction, mais un silence, une inflammation, une accélération, une poussée hors des données artistiques usuelles [...], pour migrer du côté de l'image et de ses annexes ${ }^{10}$ ". La remarque pourrait sans doute s'appliquer à bien des situations, mais ce jour-là, Élisabeth Lebovici nous parlait notamment des "désastres insurpassables ${ }^{11}$ " de l'histoire, et du sida en particulier. Que les choses soient dites, pour être claires : l'ouvrage qu'elle 


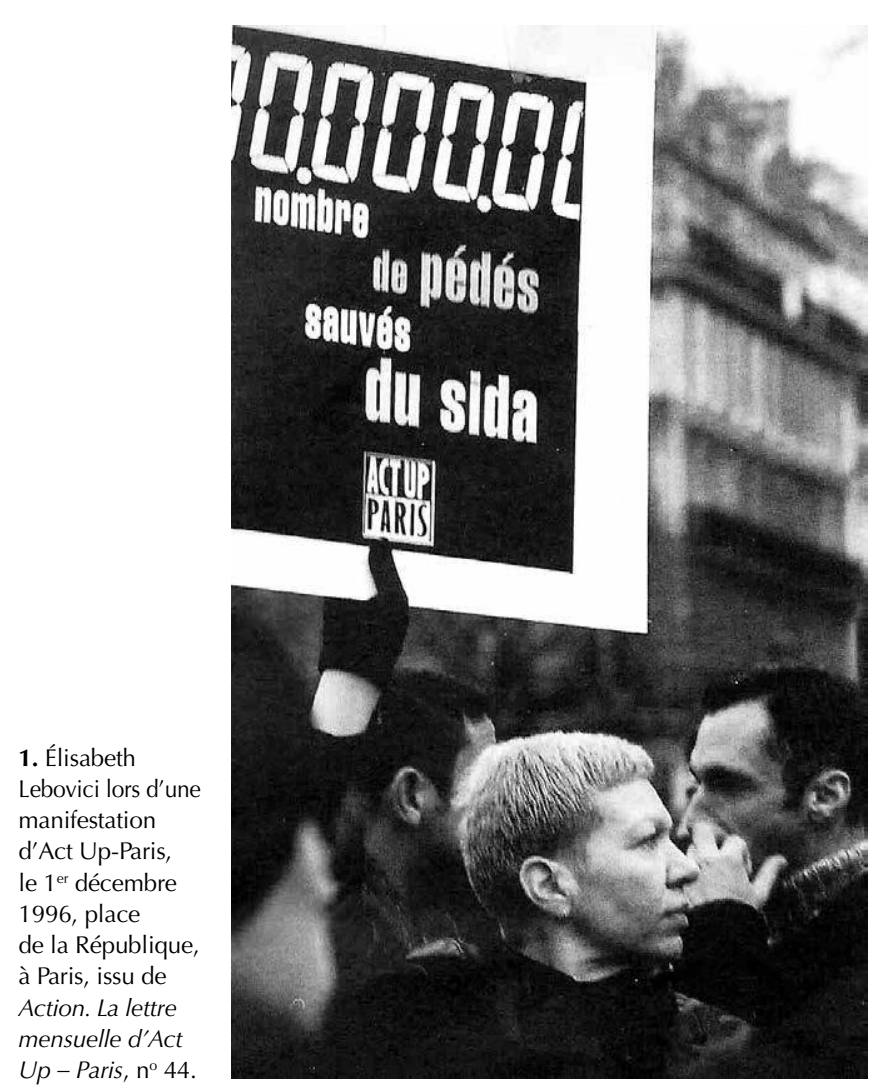

nous livre aujourd'hui n'a été ni facile à décider, ni à composer, et encore moins, sans doute, à achever, et d'abord parce que l'histoire qu'elle nous raconte ne l'est pas. "Nombre d'entre les corps amis qui m’ont accompagnée, aimée, avec qui j'ai correspondu, conversé, partagé ce que je voyais ont disparu. Je me retrouve, plus de trente ans après, à vouloir décrire ce que la spectatrice survivante a vécu ${ }^{12}$. " On comprend alors l'hommage que l'auteure rend, dès les premières lignes, à la philosophe Vinciane Despret, qui pense le dialogue avec les absents ${ }^{13}$, comme son parti pris, affirmé un peu plus loin, de la "sentimentalité ${ }^{14}$ ", sans laquelle même l'histoire et ceux qui la font sont vraiment morts.

\section{La part de l'émotion ${ }^{15}$}

À cet égard, avec Ce que le sida $m^{\prime}$ a fait, Élisabeth Lebovici s'inscrit dans une pratique de l'écriture historique qui rappelle beaucoup celle d'Arlette Farge, qui n'a jamais pensé que " l'émotion est une stérilisation de la pensée, [...] et une façon condamnable d'entrer en empathie avec l'objet de la recherche ${ }^{16} "$ - il faut dire que cette dernière a connu et travaillé avec Michel Foucault, et perçu chez lui cette "vibration physique, l'intensité des émotions, la surprise et l'effroi ${ }^{17}$ " qu'il affirmait éprouver pour ces "vies brèves ${ }^{18}$ " rencontrées au hasard de sa fréquentation des archives judiciaires. Une sismographie, encore. Ce que le sida m'a fait nous incite de nouveau à penser l'historicité des sentiments, des émotions, qui font événement dans l'histoire. "Une même sentimentalité, me semble-t-il, se manifeste dans l'archivage, non seulement des objets matériels et/ou textuels, mais des affects "associés à la nostalgie, à la mémoire personnelle, au fantasme, au traumatisme". Ce mélange dans une même matérialité nous renvoie à l'objet de ce livre ; son objet principal, pourrais-je dire, tant ce livre essaie de faire avec ces choses matérielles que sont les artefacts, mais aussi avec ces choses immatérielles que sont les sentiments - mes sentiments -, considérés comme "trop personnels et trop éphémères pour qu'on les consigne par écrit". L'objet de ce livre, $c^{\prime}$ est de ne pas faire la différence ${ }^{19}$. "

Tout est donc ici cousu ensemble, parce qu'il en est ainsi dans la vie, et Ce que le sida m'a fait, qu'elle n'aurait certainement pas pu écrire autrement, se clôt d'ailleurs sur une photographie de l'auteure prise le $1^{\text {er }}$ décembre 1996, place de la République, lors d'une manifestation d'Act UpParis ; on la voit brandir une pancarte qui évalue à zéro le nombre de "pédés sauvés du sida " (fig. 1). Bilan tragique de l'incurie des pouvoirs, qui ramène, comme beaucoup d'autres images - qui " n'illustrent " pas, mais dialoguent dans et avec le texte - à ces "vies interrompues", au " manque ", au "désespoir ", à la "rage ", au " temps condensé ", à la "vitalité " aussi, ou encore aux amitiés, aux amours, au "désir ${ }^{20}$ ". Alors les indices d'une "inflammation ", d'une " accélération ", d'une " poussée " ne sont pas seulement à chercher du côté de l'image élargie, qui dérange nos habitudes visuelles, mais également de ce qui travaille l'auteure en souterrain, fait se heurter ses mémoires, remonter à la surface ses " obsessions ${ }^{21}$ ": " "l'achèvement, l'inachèvement, l'interminable", la péremption, l'aprèscoup, les "machines affectées", le "fragment", "des corps dans le décor", "l'intime", etc. ${ }^{22}$ " Elles produisent un récit qui ne prétend pas, en seize chapitres, à l'exhaustivité, mais s'attache aux êtres et aux choses, aux événements, c'est-à-dire à tout ce qui a semblé important à Élisabeth 


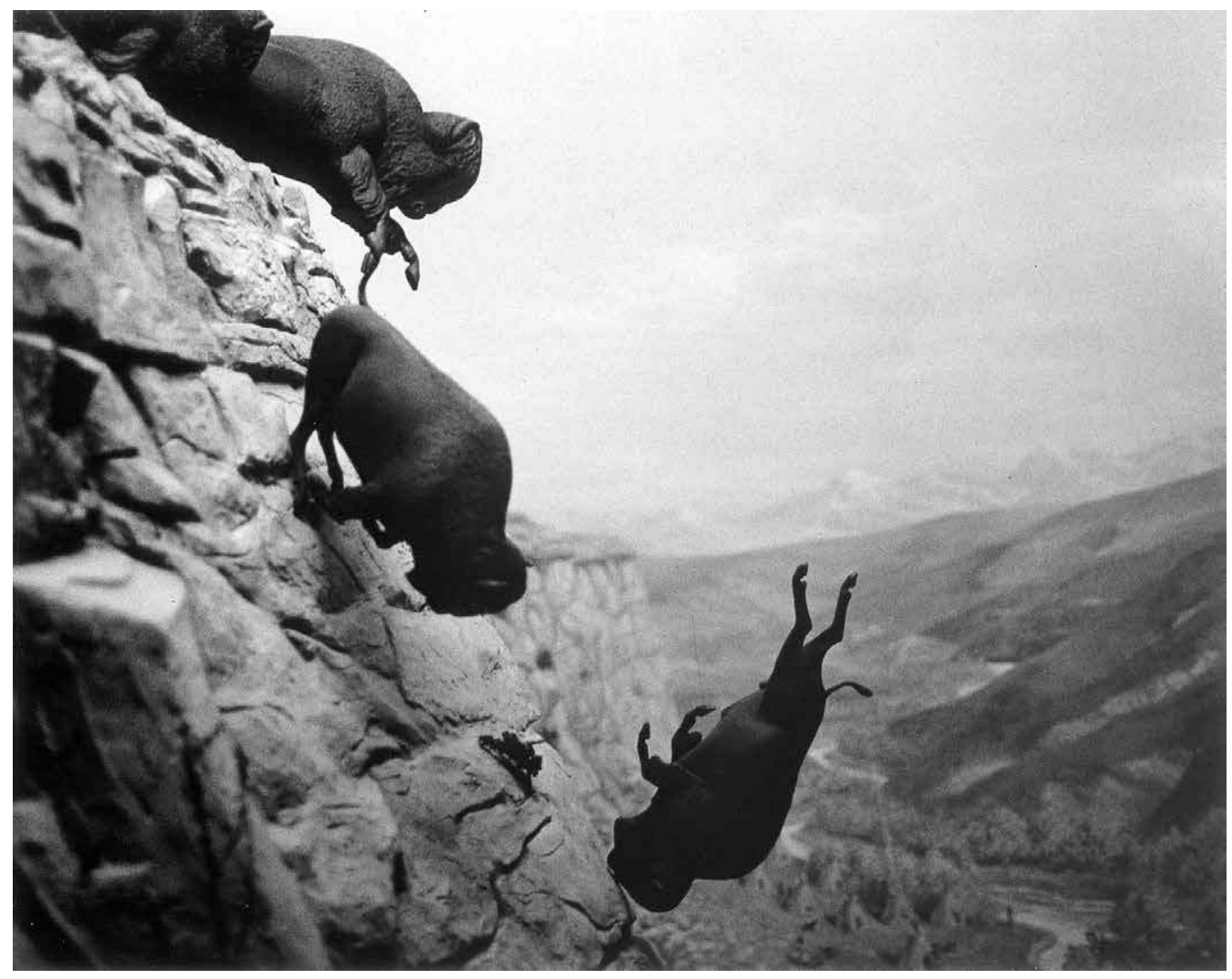

2. David

Wojnarowicz, Untitled (Buffalo),

1988-1989 [tirage de 1994], épreuve gélatinoargentique, New York, PPOW Gallery.
Lebovici lorsqu'il s'est agi de rassembler, pour chaque chapitre, d'anciens textes - souvent des versions françaises d'essais publiés en anglais, puis de les remanier largement. Tout ce qui devait être là, se rencontrer, coexister - à nouveau, encore. En choisissant subjectivement de qui ou de quoi elle veut nous parler, l'auteure opère un déplacement dans la construction même d'une histoire qui refuse volontairement l'exhaustivité au profit de "l'anecdote ", celle-ci redéfinissant le rapport entre celle qui écrit, ce qu'elle écrit et le lecteur, en y inscrivant des affects, précisément. Voilà le lecteur prévenu : Ce que le sida m'a fait " n'est ni une compilation des morts ni un catalogue des figurations artistiques du VIH/sida ${ }^{23}$. "

\section{Outre-Atlantique}

Rien à voir, en l'état, avec Art AIDS America, l'épais catalogue de près de trois cents pages dirigé par Jonathan David Katz ${ }^{24}$ et Rock Hushka et publié à l'occasion de l'exposition éponyme ${ }^{25}$. Si, en France, aucune exposition exclusivement consacrée aux représentations de la séropositivité et du sida en art n'a jamais été organisée, outre-Atlantique, un certain nombre d'expositions l'ont été26, sans toutefois recouvrir l'ampleur d'Art AIDS America, où pour la première fois la réunion de près de cent trente œuvres ambitionnait d'offrir un panorama complet de la création artistique américaine sur la question.

Une quinzaine d'auteurs ont collaboré au catalogue, pour démontrer l'hypothèse séminale de Katz : "le sida a changé l'art américain ${ }^{27}$ ". $C^{\prime}$ est le sens du titre qu'Élisabeth Lebovici a donné à son ouvrage : "Ce que le sida $m^{\prime} a$ fait. J'ai souhaité personnaliser le titre [...]. Mais derrière ce "moi", il n'y a pas que moi, même si je parle de mon expérience et même s'il me semble toujours que ce "discours à la première personne" est un enjeu crucial aussi bien qu'un héritage de l'épidémie. Mais derrière ce " $m$ ", il y a l'art. Il y a des expositions. Il y a des pratiques artistiques. Des pratiques curatoriales. Des discours critiques. Une géographie. Comme il y a des façons de regarder, de se situer, de prendre et de donner ${ }^{28}$." Ce que le sida a fait à l'art : voilà qui ramène à l'histoire d'un tremblement et bien plus encore, 
3. Izhar Patkin, Unveiling of a Modern Chastity, 1981 caoutchouc, latex et encre sur toile.

\section{Felix} Gonzalez-Torres, Untitled (Himmler, Hate, Hole, Helms), 1990, vue de l'exposition "Collector", Lille, TriPostal (5 octobre 2011 $-1^{\text {er }}$ janvier 2012), Centre national des arts plastiques, FNAC 94035.

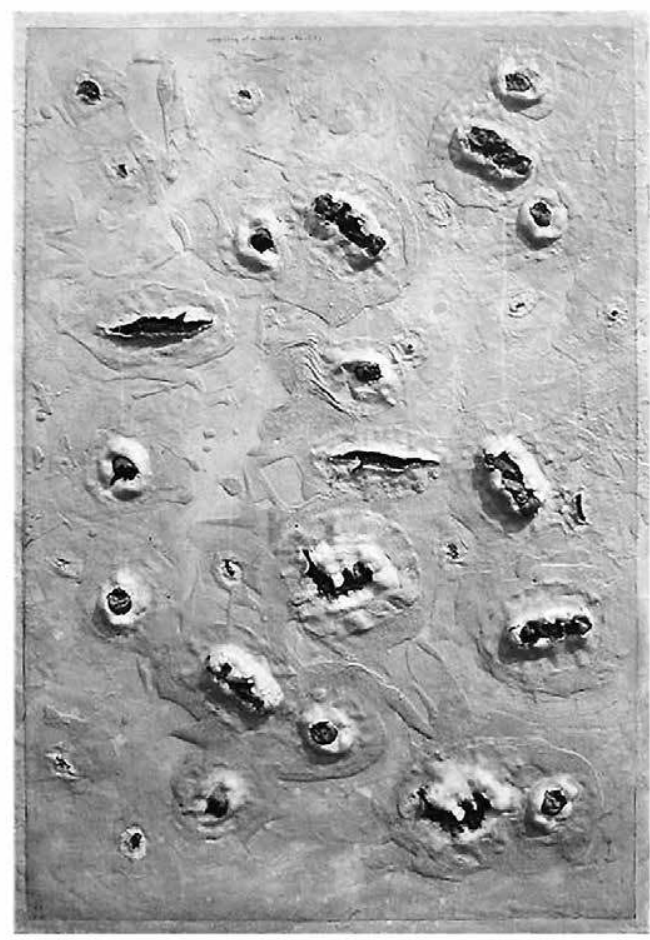

lorsque le sol même s'est dérobé sous les pieds d'une génération, à une faille jamais refermée (fig. 2). D'un ouvrage à l'autre, la même référence à la " mort de l'auteur " annoncée par Roland Barthes, devenue, à la fin des années 1960, la condition de "la naissance du lecteur ${ }^{29}$ ", et cette même sidération quand, avec le sida, l'hypothèse théorique est devenue réalité. Élisabeth Lebovici et Jonathan D. Katz les partagent, comme d'ailleurs l'expérience de l'activisme contre le sida et pour les luttes homosexuelles, et la première revendique de porter l'accent dans son ouvrage sur les lesbiennes, longtemps grandes oubliées de l'épidémie ${ }^{30}$, le second invite des figures de combat dans le catalogue d'Art AIDS America. C'est peu dire qu'ils ont été les acteurs de l'histoire qu'ils nous livrent aujourd'hui, ce qui, du point de vue de son écriture - de témoignage, en partie - n'a bien sûr rien d'anodin. Ils n'avaient pas trente ans lorsque sont parus les premiers articles sur ce que l'on appelait alors le " cancer gay ". C'était en 1981 et, cette année-là, Izhar Patkin, un jeune artiste israélien installé à New York depuis 1979 pour suivre lui aussi le Whitney Independent Study Program, donnait une première forme libre à son angoisse dans un cauchemar prémonitoire de caoutchouc et de latex (fig. 3).

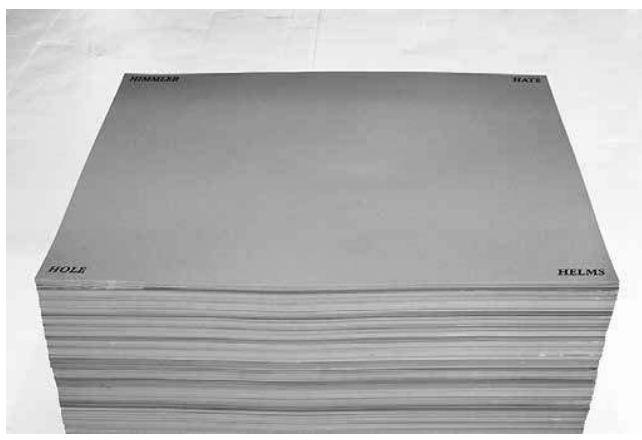

Unveiling of a Modern Chastity a figuré dans Art AIDS America et dans son catalogue, quand bien même l'œuvre, qui venait alors de sortir de l'atelier de Patkin après trente années de réclusion, a pu déranger quelques-unes des thèses de Jonathan D. Katz. Écartant presque tout l'activisme culturel, ce qui constituait déjà un parti pris très fort, le concept qui sous-tendait Art AIDS America se fondait en effet sur un corpus d'œuvres qui déjouaient la censure de l'époque en adoptant les formes codées et acceptables qui devaient garantir qu'elles seraient accueillies dans le monde de l'art ; ainsi celles du minimalisme, reconduites et subverties notamment par Felix Gonzalez-Torres (fig. 4). Mais par sa précocité, l'œuvre de Patkin oppose la difficulté de la resituer dans un dialogue avec le discours sur la maladie tel qu'il s'est développé après 1981, d'où Jonathan D. Katz fait naître la réponse artistique à la crise. Aux premières heures de l'épidémie, elle n'a en effet pas fonctionné dans ce système " métaphorique " que l'historien de l'art qualifie de "postmodernisme poétique ".

C'est le problème de tout système, qui n'aime rien tant qu'être rassuré dans sa rationalité et, pour cela, fonctionne souvent en circuit fermé. Ainsi Art AIDS America prenait-elle également le parti de ne se concentrer que sur l'art américain, ce qui se justifie à certains égards, mais réduit considérablement le spectre de l'analyse - comme celui d'un "grand récit " blanc/gay/masculin pour qui se propose de " faire du sida un acteur actif de l'histoire " et de " comprendre qu'elle [l'épidémie] est en réalité notre histoire, un traumatisme collectif, ainsi que son impact global ${ }^{31}$ ".

Élisabeth Lebovici, quant à elle, ne s'est pas épargné l'effort de penser la discussion transatlantique, peut-être parce qu'elle n'est jamais vraiment revenue de New York, surtout parce que son panthéon visuel et intellectuel est autant américain 
qu'européen, où Judith Butler côtoie Monique Wittig, Roland Barthes Douglas Crimp, etc., et qu'elle sait combien tout a communiqué dans - et malgré - la crise du sida, et jusqu'aux formes mêmes qu'elle provoquait. Voilà qui atteste amplement de l'extraordinaire puissance de dérangement de l'épidémie dans les vies, de part et d'autre de l'Atlantique, et perturbe assurément une certaine histoire de l'art qui peine encore à la reconnaître. $C^{\prime}$ 'est vrai aux États-Unis, où les commissaires d'Art AIDS America ont eu beaucoup de difficultés à imposer leur projet, encore en 2015 ; c'est d'autant plus vrai en France, où la sortie de Ce que le sida m'a fait est un événement au regard de la nouveauté et de l'incitation à la recherche qu'il représente, tant sont rares les historiens de l'art à s'être vraiment intéressés au sida et à ses représentations.

\section{Essayer de tout (re)penser}

Il a ainsi fallu attendre 2016, presque une éternité, pour que soient traduits en français les écrits du critique d'art et militant homosexuel américain Douglas Crimp ${ }^{32}$. Figure majeure du discours sur le sida, auquel il a si puissamment contribué, comme de la théorie queer qui s'y fonde en partie, Crimp a marqué l'ensemble des auteur.e.s qui nous intéressent ici. Assurément, leur dette à son égard est immense, et pour cause.

À l'hiver 1987 est paru le quarante-troisième numéro d'October (fig. 5) qu'il a dirigé et entièrement consacré au sida : " AIDS: Cultural Analysis/ Cultural Activism ", le plus gros numéro de la revue jamais publié (273 pages, 14 essais), le plus vendu également, et le seul réédité sous forme de livre $^{33}-c^{\prime}$ est dire son importance. Universitaires et militants y pensaient ensemble l'épidémie du sida dans ses implications culturelles et les usages politiques de ses représentations, ce qui était neuf à l'époque. Contre l'apathie du monde de l'art et sa politique du chef-d'œuvre, Crimp ouvrait grand en effet le champ de la réflexion, convaincu que les réponses activistes à la crise devaient aussi y jouer un rôle essentiel. Dans son introduction, il résumait ce déplacement fondateur de la pratique activiste : "L'art a le pouvoir de sauver des vies, et c'est précisément ce pouvoir qui doit être reconnu, nourri et soutenu par tous les moyens. Nous n'avons nul besoin de renaissance culturelle; nous avons besoin de pratiques culturelles prenant activement part à la lutte contre le sida. Nous n'avons nul besoin de transcender l'épidémie ; nous avons besoin d'y mettre un terme ${ }^{34}$."

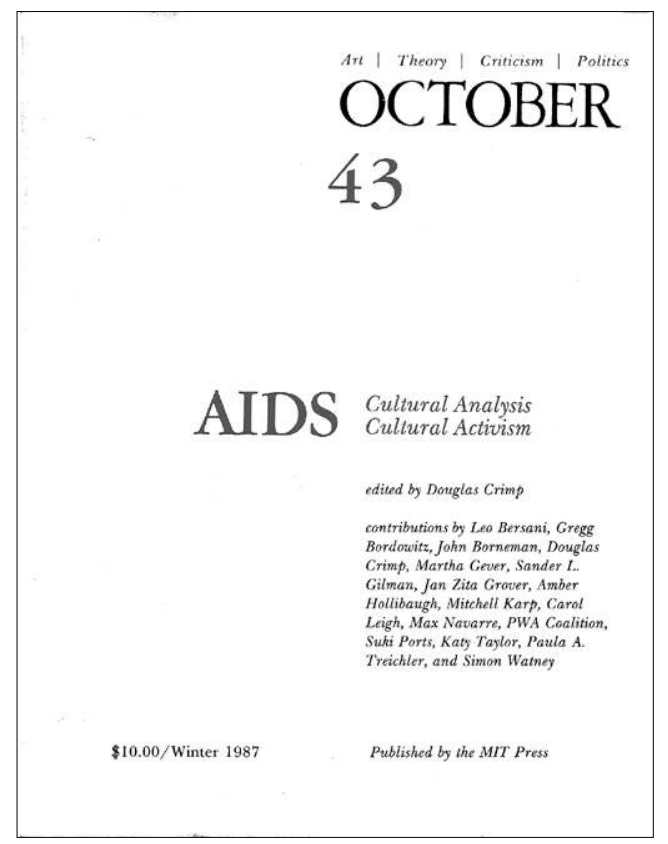

5. Première de couverture de « AIDS. Cultural Analysis/Cultural Activism ", October, n ${ }^{\circ} 43$, hiver 1987.

Lors des émeutes de Stonewall, en juin 1969 , Crimp avait vingt-cinq ans, et âgé de quarante-trois ans à la sortie d' " AIDS: Cultural Analysis/Cultural Activism ", il comprenait combien le sida rebattait brutalement les cartes du jeu social. Auparavant, dans l'entourage de Rosalind Krauss, Craig Owens et d'autres, il avait contribué à conceptualiser le "postmodernisme " aux États-Unis, autour de la "Pictures Generation ", notamment contre la doxa moderniste de l'autonomie de l'œuvre d'art. Mais en 1987 son ami le poète, artiste et activiste Gregg Bordowitz, qui livra lui aussi un article pour "AIDS: Cultural Analysis/Cultural Activism ", l'emmenait aux réunions hebdomadaires d'Act UpNew York, et Crimp allait bientôt renoncer à la critique d'art pour le combat en faveur du maintien de la diversité des cultures sexuelles et identitaires. Car là, selon lui, se jouait vraiment l'époque, avec laquelle il changea, comme ses outils pour la penser.

Il faut bien comprendre tout ce que le sida a fait perdre à ceux d'une génération qui se sont (re-)trouvés dans l'œil noir de sa tempête : les êtres, d'abord, qui comptent toujours plus que le reste, et pour " ceux qui se proposent d'essayer de penser ", comme disait Gilles Deleuze, jusqu'à leurs repères. Ce que le sida a fait, c'est les confronter au vide : " décimées par l'épidémie, ruinées par le deuil, cassées dans la peur et la mélancolie ", écrit Élisabeth Lebovici, " une génération entière d'historien.ne.s de l'art comme une génération 
de pratiques, non seulement artistiques mais critiques, ont manqué ${ }^{35}$ ". L'auteure pense sans doute, entre beaucoup d'autres, à son cher ami Patrick Bracco, spécialiste des jardins et des feux d'artifices $^{36}$, mort en 1986, et sa remarque vaut pour Crimp notamment, qui dans les années 1980 a reçu comme un choc les morts cumulées de Michel Foucault (1984), un phare, et de son ami Guy Hocquengehm (1988), le " philosophe du désir homosexuel ${ }^{37}$ ". Il fallait désormais vivre dans cette proximité avec la catastrophe, à partir de là - vivre sans, et avec. À trente ans, au sortir d'un autre désastre, T. S. Eliot circulait sur sa Terre vaine pour, $\mathrm{d}^{\prime}$ " images brisées » et de " fragments ", " étayer [ses] ruines ". Trouver autre chose, qui, dans l'effondrement, rendrait le monde moins inhabitable, non pas un substitut, mais une forme de réinvention, et même du temps renversé quand la mort a le visage de la jeunesse volée. Cela a valu pour les artistes, au sens très large du mot, et les ouvrages dont il est ici question le démontrent amplement, comme pour ceux qui les fréquentent et en parlent, qui ont également dû modifier leur outillage critique. À cet égard, le parcours de Crimp, passé du comité de rédaction d'October aux réunions d'Act Up-New York est exemplaire d'un dessaisissement de ce qui, dans la crise, ne tenait plus à ses yeux : une certaine manière de penser - de faire (de) - l'histoire de l'art, et cette sortie de la discipline et de ses règles comme de soi, aussi une levée d'autocensure, est un autre indice de l'énorme crise de représentation qui s'ouvrait alors.

Et l'on comprend, à lire ensemble le recueil qu'il coordonna en 1987, Art AIDS America et Ce que le sida m'a fait, combien dans la crise épidémique, jamais refermée, les manières de regarder et d'écrire ne pouvaient définitivement plus être les mêmes dès lors qu'elle avait tout emporté, combien l'engagement ouvrait - forçait à s'ouvrir à de nouveaux corpus, méthodes et savoirs à partir desquels réinvestir le champ des arts et tout repenser, et d'abord une définition élargie de l'activisme qui opère à tous les niveaux du privé et du public. Depuis ces territoires, et Act Up-Paris notamment, Élisabeth Lebovici a ainsi éprouvé la validité de la proposition d'Eve Kosofsky Sedgwick, selon laquelle "l'homophobie est misogyne " - elle la relit d'ailleurs sous la plume de Leo Bersani, en 1987 dans " AIDS: Cultural Analysis/Cultural Activism " -, et beaucoup de ce qu'elle est et de ce qu'elle fait aujourd'hui, de ce qu'elle nous donne à lire et penser vient de là, jusqu'à son dernier magnum opus sur le sida, précisément sous-titré Art et activisme à la fin du $X X^{e}$ siècle $^{38}$.

Des terribles et mal nommées " années sida ", il faut se souvenir de tout pour ne plus rien en perdre, à plus forte raison parce que cette histoire n'est pas achevée. Il faut se souvenir de tout, et notamment de ce qu'elles firent à l'art, de leurs « images malades ", et de ceux qui prirent le risque de les regarder en face. Ils nous apprennent à leur tour quelque chose, et sur la fonction sociale de l'art, évidemment, sa centralité en politique, mais encore sur les conditions de la pratique de la critique d'art et de l'histoire de l'art, qui ne peut pas, ne doit pas être froidement figée et neutre. Au même titre que les artistes et leurs œuvres, elle sait et peut agir sur l'histoire, s'y engager pour y jouer un rôle à proportion de sa capacité et de sa puissance à saisir les enjeux de son temps, et à les formuler. Dans nos inquiétudes et nos régressions actuelles, sans doute avons-nous besoin d'en être (r)assurés.

\section{Thibault Boulvain,}

université Paris 1 Panthéon-Sorbonne thibault.boulvain@club-internet.fr 
1. Cité par Alain Corbin, dans Alain Corbin, Gilles Heuré, Historien du sensible. Entretien avec Gilles Heuré, Paris (collection Cahiers libres), 2000, p. 127.

2. LEBOVICI, 2017, p. 10.

3. Ibidem, p. 90.

4. Ibid., p. 187, je souligne.

5. Ibid., p. 200.

6. Ibid., p. 134.

7. Titre de sa thèse de doctorat en esthétique, soutenue à l'université Paris 10 Nanterre, en 1983.

8. Lebovici, 2017, p. 136.

9. Ibid., p. 10 .

10. Communication délivrée à l'occasion de "Let's Queer Art History! ", conférence, 21 mai 2001, organisée dans le cadre de "Selon Patricia Falguières. L'histoire de l'art par ses artistes, même ", cycle de conférences du 28 avril au 26 mai 2011, organisé par Patricia Falguières, Centre Pompidou, Paris.

11. Voir la note précédente.

12. LeBOVICI, 2017, p. 134.

13. Vinciane Despret, Au bonheur des morts. Récits de ceux qui restent, Paris (collection Les Empêcheurs de penser en rond), 2015. LEBOVICI, 2017, p. 9.

14. Ibid., p. 13.

15. J'emprunte ce titre à Arlette Farge, "La part de l'émotion ", dans Socio-anthropologie, $\mathrm{n}^{\circ}$ 27, 2013, p. 99-101.

16. Arlette Farge, "Histoire, événement, parole ", dans Socio-anthropologie, $\mathrm{n}^{\circ} 2,1997$, mis en ligne le 15 janvier 2003, http://socio-anthropologie.revues.org/29 (consulté le 24 mars 2017).

17. Mario Colucci, «Hystériques, internés, hommes infâmes : Michel Foucault et la résistance au pouvoir ", dans Alain Beaulieu (dir.), Michel Foucault et le contrôle social, Laval (collection Mercure du Nord), 2005, p. 86.

18. Michel Foucault, "La vie des hommes infâmes ", dans Cahiers du chemin, $\mathrm{n}^{\circ} 29,1977$, repris dans Dits et écrits, Paris, 1994, p. 239.

19. LeBovici, 2017, p. 13.

20. Ibid., p. 10.

21. Ibid.

22. Ibid.

23. Ibid., p. 9.

24. David Katz (Director, PhD Program in Visual Studies, State University of New York, Buffalo) a auparavant été le commissaire, avec David C. Ward, d' « Hide/Seek. Difference and Desire in American Portraiture ", National Portrait Gallery, Smithsonian Institution, Washington, 30 octobre 2010 - 13 février 2011.

25. Rock Hushka est Chief Curator au Tacoma Art Museum. "Art AIDS America ", Tacoma Art Museum, Tacoma, 3 octobre 2015 - 10 janvier 2016 ; Zuckerman Museum of Art, Kennesaw, 20 février - 22 mai 2016 ;
The Bronx Museum of the Arts, New York, 13 juillet - 25 septembre 2016 ; Alphawood Gallery, Chicago, $1^{\text {er }}$ décembre $2016-2$ avril 2017.

26. On pense notamment à l'une des premières, majeure : "Witnesses: Against Our Vanishing ", organisée par Nan Goldin à l'Artists Space, New York, 16 novembre 1989 - 6 janvier 1990. Élisabeth Lebovici y consacre le chapitre X de son ouvrage (LEBOVICI, 2017, p. 225-237).

27. "How AIDS Changed American Art " est le titre de l'essai introductif de Jonathan David Katz, dans Art AIDS America, 2015, p. 24-45.

28. Lebovici, 2017, p. 9

29. Roland Barthes, "La mort de l'auteur ", dans Euvres complètes, II, 1966-1973, Éric Marty (éd.), Paris, 1994, p. 495.

30. Lebovici, 2017, p. 12.

31. Katz, 2015, cité n. 27, p. 24.

32. Douglas Crimp, Gaëtan Thomas (dir.), PICTURES. S'approprier la photographie. New York, 1979-2014, Nicolas Paul, Gaëtan Thomas (trad. fra.), Paris, 2016.

33. CRIMP, 1988.

34. Ibid., p. 7.

35. LEBOVICI, 2017, p. 39-40.

36. On lui doit notamment, en collaboration avec Élisabeth Lebovici, Fireworks/Feux d'Artifices, French Fireworks from the 17th to the 19th Century, cat. exp. (Washington, DC, National Gallery of Art, 1976), Washington, 1976 ; Feux d'artifice et illuminations sous le Premier Empire, Patrick Bracco, Élisabeth Lebovici (dir.), cat. exp. (Boulogne-Billancourt, bibliothèque Marmottan, 1977), Boulogne-Billancourt, 1977 ; Patrick Bracco, Élisabeth Lebovici, Ruggieri, 250 ans de feux d'artifice, Paris, 1988.

37. LEBOVICI, 2017, p. 197.

38. On la connait notamment pour ses nombreuses contributions relatives au féminisme, aux questions de genre et à la théorie queer, ainsi : Élisabeth Lebovici (dir.), L'intime, Paris, 1998 ; avec Catherine Gonnard, Femmes artistes/artistes femmes : Paris, de 1880 à nos jours, Paris, 2007. Elle est membre du groupe de recherches "Travelling Féministe ", autour des archives du Centre audiovisuel Simone de Beauvoir et membre fondatrice du Fonds de dotation " Lesbiennes d'intérêt général " (LIG). 